\title{
Equal Ratio Gain Technique and Its Application in Linear General Integral Control
}

\author{
Baishun Liu \\ Academy of Naval Submarine, Qingdao, China \\ Email: baishunliu@163.com \\ Received 30 September 2014; accepted 13 October 2014; published 27 February 2015 \\ Copyright (C) 2015 by author and Scientific Research Publishing Inc. \\ This work is licensed under the Creative Commons Attribution International License (CC BY). \\ http://creativecommons.org/licenses/by/4.0/

(c) (i) Open Access

\section{Abstract}

In conjunction with linear general integral control, this paper proposes a fire-new control design technique, named Equal ratio gain technique, and then develops two kinds of control design methods, that is, Decomposition and Synthetic methods, for a class of uncertain nonlinear system. By Routh's stability criterion, we demonstrate that a canonical system matrix can be designed to be always Hurwitz as any row controller gains, or controller and its integrator gains increase with the same ratio. By solving Lyapunov equation, we demonstrate that as any row controller gains, or controller and its integrator gains of a canonical system matrix tend to infinity with the same ratio, if it is always Hurwitz, and then the same row solutions of Lyapunov equation all tend to zero. By Equal ratio gain technique and Lyapunov method, theorems to ensure semi-globally asymptotic stability are established in terms of some bounded information. Moreover, the striking robustness of linear general integral control and PID control is clearly illustrated by Equal ratio gain technique. Theoretical analysis, design example and simulation results showed that Equal ratio gain technique is a powerful tool to solve the control design problem of uncertain nonlinear system.

\section{Keywords}

Equal Ratio Gain Technique, General Integral Control, Nonlinear Control, Robust Control, Output Regulation

\section{Introduction}

The complexity of nonlinear system challenges us to come up with systematic design methods to meet control objectives and specifications. Faced with such challenge, it is clear that we can not expect a particular method to 
apply to all nonlinear systems [1]. Therefore, although there were Linearization techniques, Gain scheduling technique, Singular perturbation technique, feedback linearization technique, sliding mode technique and so on, nonlinear design tools, this paper still develops a new control design technique, named Equal ratio gain technique, in conjunction with linear general integral control since integral control plays an irreplaceable role in the control domain.

For general integral control design, there were various design methods, such as general integral control design based on linear system theory, sliding mode technique, Feedback linearization technique and Singular perturbation technique and so on, presented by [2]-[5], respectively. In addition, general concave integral control [6], general convex integral control [7], constructive general bounded integral control [8] and the generalization of the integrator and integral control action [9] were all developed by Lyapunov method. For illustrating the practicability and validity of Equal ratio gain technique and the good robustness of linear general integral control, this paper addresses general integral control design again.

Based on Equal ratio gain technique, this paper develops two kinds of systematic methods to design linear general integral control for a class of uncertain nonlinear system, that is, one is Decomposition method and another is Synthetic method. The main contributions are as follows: 1) a canonical system matrix can be designed to be always Hurwitz as any row controller gains, or controller and its integrator gains increase with the same ratio; 2) as any row controller gains, or controller and its integrator gains of a canonical system matrix tend to infinity with the same ratio, if it is always Hurwitz, and then the same row solutions of Lyapunov equation all tend to zero; 3) theorems to ensure semi-globally asymptotic stability are established in terms of some bounded information. Moreover, the striking robustness of linear general integral control and PID control is clearly illustrated by Equal ratio gain technique. All these mean that Equal ratio gain technique is a powerful tool to solve the control design problem of uncertain nonlinear system, and then makes the engineers more easily design a stable controller. Consequently, Equal ratio gain technique has not only the important theoretical significance but also the broad application prospects.

Throughout this paper, we use the notation $\lambda_{m}(A)$ and $\lambda_{M}(A)$ to indicate the smallest and largest eigenvalues, respectively, of a symmetric positive define bounded matrix $A(x)$, for any $x \in R^{n}$. The norm of vector $x$ is defined as $\|x\|=\sqrt{x^{T} x}$, and that of matrix $A$ is defined as the corresponding induced norm $\|A\|=\sqrt{\lambda_{M}\left(A^{\mathrm{T}} A\right)}$.

The remainder of the paper is organized as follows: Section 2 demonstrates Equal ratio gain technique. Section 3 addresses the control design. Example and simulation are provided in Section 4. Conclusions are given in Section 5.

\section{Equal Ratio Gain Technique}

Consider the following $n+1 \times n+1$ system matrix $A$,

$$
A=\left[\begin{array}{ccccc}
0 & 1 & \cdots & 0 & 0 \\
0 & 0 & \ddots & 0 & 0 \\
0 & 0 & \cdots & 1 & 0 \\
-\varepsilon_{\alpha}^{-1} \alpha_{1} & -\varepsilon_{\alpha}^{-1} \alpha_{2} & \cdots & -\varepsilon_{\alpha}^{-1} \alpha_{n} & -\varepsilon_{\alpha}^{-1} \alpha_{n+1} \\
\varepsilon_{\beta}^{-1} \beta_{1} & \varepsilon_{\beta}^{-1} \beta_{2} & \cdots & \varepsilon_{\beta}^{-1} \beta_{n} & 0
\end{array}\right]
$$

where $\alpha_{i}(i=1,2, \cdots n+1), \beta_{j}(j=1,2, \cdots n), \varepsilon_{\alpha}$ and $\varepsilon_{\beta}$ are all positive constants. If $\varepsilon_{\alpha}^{-1} \alpha_{i}$ and $\varepsilon_{\beta}^{-1} \beta_{j}$ are viewed as the controller and integrator gains, respectively, and then the system matrix $A$ is an $n+1$-order single input single output linear system matrix with linear general integral control. In consideration of the controllable canonical form of linear system, the system matrix $A$ can be called as the controllable canonical form with linear general integral control.

For developing Equal ration gain technique, firstly, we must ensure that the system matrix $A$ is Hurwitz for all $0<\varepsilon_{\alpha}<\varepsilon_{\alpha}^{*}$ and $0<\varepsilon_{\beta}<\varepsilon_{\beta}^{*}$, secondly we need to solve the Lyapunov equation $P A+A^{\mathrm{T}} P=-Q$ with any given positive define symmetric matrix $Q$ to obtain the solution of the matrix $P$. 


\subsection{Hurwitz Stability}

For $0<\varepsilon_{\alpha}<\varepsilon_{\alpha}^{*}$ and $0<\varepsilon_{\beta}<\varepsilon_{\beta}^{*}$, Hurwitz stability of the system matrix $A$ can be achieved by Routh's stability criterion, as follows:

Step 1: the polynomial of the system matrix $A$ with $\varepsilon_{\alpha}=\varepsilon_{\beta}=1$ is,

$$
s^{n+1}+\alpha_{n} s^{n}+\left(\alpha_{n+1} \beta_{n}+\alpha_{n-1}\right) s^{n-1}+\cdots+\left(\alpha_{n+1} \beta_{2}+\alpha_{1}\right) s+\alpha_{n+1} \beta_{1}=0
$$

By Routh's stability criterion, the gains $\alpha_{i}$ and $\beta_{j}$ can be chosen such that the polynomial (1) is Hurwitz. Obviously, if $\alpha_{i}$ and $\beta_{j}$ are all large to zero, and then the necessary condition, that is, the coefficients of the polynomial (1) are all positive, is naturally satisfied.

Step 2: based on the gains $\alpha_{i}, \beta_{j}$ and Hurwitz stability condition to be obtained by Step 1, the maximums of $\varepsilon_{\alpha}$ and $\varepsilon_{\beta}$, that is, $\varepsilon_{\alpha}^{*}$ and $\varepsilon_{\beta}^{*}$, can be obtained, respectively. Since $\varepsilon_{\alpha}$ and $\varepsilon_{\beta}$ interact, there exist innumerable $\varepsilon_{\alpha}^{*}$ and $\varepsilon_{\beta}^{*}$, but $\varepsilon_{\alpha}^{*}$ is more important than $\varepsilon_{\beta}^{*}$. Thus, two kinds of typical cases are interesting, that is, one is that $\varepsilon_{\alpha}^{*}$ is evaluated with $\varepsilon_{\beta}=1$; another is that let $\varepsilon_{\alpha}=\varepsilon_{\beta}$, and then $\varepsilon_{\alpha}^{*}=\varepsilon_{\beta}^{*}$ can be obtained together.

Step 3: by $\varepsilon_{\alpha}^{*}$ and $\varepsilon_{\beta}^{*}$ obtained by Step 2, check Hurwitz stability of the system matrix $A$ for all $0<\varepsilon_{\alpha}<\varepsilon_{\alpha}^{*}$ and $0<\varepsilon_{\beta}<\varepsilon_{\beta}^{*}$. If it does not hold, redesign $\alpha_{i}$ and $\beta_{j}$ and repeat the previous steps until the system matrix $A$ is Hurwitz for all $0<\varepsilon_{\alpha}<\varepsilon_{\alpha}^{*}$ and $0<\varepsilon_{\beta}<\varepsilon_{\beta}^{*}$.

The demonstration above is only a basic idea to ensure that the system matrix $A$ is Hurwitz. For clearly illustrating the method above, we consider two kinds of cases, that is, $n=2$ and $n=3$, respectively, as follows:

Case 1: for $n=2$, the polynomial (1) is,

$$
s^{3}+\alpha_{2} s^{2}+\left(\alpha_{3} \beta_{2}+\alpha_{1}\right) s+\alpha_{3} \beta_{1}=0
$$

By Routh's stability criterion, if $\alpha_{3}, \alpha_{2}, \alpha_{1}, \beta_{2}$, and $\beta_{1}$ are all positive constants, and the following inequality,

$$
\alpha_{2}\left(\alpha_{3} \beta_{2}+\alpha_{1}\right)>\alpha_{3} \beta_{1}
$$

holds, and then the polynomial (2) is Hurwitz.

Sub-class 1: $\alpha_{3}, \alpha_{2}$ and $\alpha_{1}$ are multiplied by $\varepsilon_{\alpha}^{-1}$, and then substituting them into (3), obtain,

$$
\alpha_{2}\left(\alpha_{3} \beta_{2}+\alpha_{1}\right)>\varepsilon_{\alpha} \alpha_{3} \beta_{1}
$$

By the inequality (4), obtain,

$$
\varepsilon_{\alpha}^{*}=\frac{\alpha_{2}\left(\alpha_{3} \beta_{2}+\alpha_{1}\right)}{\alpha_{3} \beta_{1}}
$$

Sub-class 2: $\alpha_{3}, \alpha_{2}, \alpha_{1}, \beta_{2}$ and $\beta_{1}$ are multiplied by $\varepsilon_{\alpha}^{-1}$, and then substituting them into (3), obtain,

$$
\alpha_{2} \alpha_{3} \beta_{2}>\varepsilon_{\alpha}\left(\alpha_{3} \beta_{1}-\alpha_{2} \alpha_{1}\right)
$$

For this sub-class, there are two kinds of cases:

1) if $\alpha_{3} \beta_{1}-\alpha_{2} \alpha_{1}>0$, and then by the inequality (5), obtain,

$$
\varepsilon_{\alpha}^{*}=\frac{\alpha_{2} \alpha_{3} \beta_{2}}{\alpha_{3} \beta_{1}-\alpha_{2} \alpha_{1}}
$$

2) if $\alpha_{3} \beta_{1}-\alpha_{2} \alpha_{1} \leq 0$, and then by the inequality (5), obtain,

$$
\varepsilon_{\alpha}^{*}=\infty
$$

Case 2: for $n=3$, the polynomial (1) is,

$$
s^{4}+\alpha_{3} s^{3}+\left(\alpha_{4} \beta_{3}+\alpha_{2}\right) s^{2}+\left(\alpha_{4} \beta_{2}+\alpha_{1}\right) s+\alpha_{4} \beta_{1}=0
$$

By Routh's stability criterion, if $\alpha_{4}, \alpha_{3}, \alpha_{2}, \alpha_{1}, \beta_{3}, \beta_{2}$ and $\beta_{1}$ are all positive constants, and the fol- 
lowing inequality,

$$
\alpha_{3}\left(\alpha_{4} \beta_{3}+\alpha_{2}\right)\left(\alpha_{4} \beta_{2}+\alpha_{1}\right)>\left(\alpha_{4} \beta_{2}+\alpha_{1}\right)^{2}+\alpha_{3} \alpha_{3} \alpha_{4} \beta_{1}
$$

holds, and then the polynomial (6) is Hurwitz.

Sub-class 1: $\alpha_{4}, \alpha_{3}, \alpha_{2}$ and $\alpha_{1}$ are multiplied by $\varepsilon_{\alpha}^{-1}$, and then substituting them into (7), obtain,

$$
\alpha_{3}\left(\alpha_{4} \beta_{3}+\alpha_{2}\right)\left(\alpha_{4} \beta_{2}+\alpha_{1}\right)-\alpha_{3} \alpha_{3} \alpha_{4} \beta_{1}>\varepsilon_{\alpha}\left(\alpha_{4} \beta_{2}+\alpha_{1}\right)^{2}
$$

By the inequality (8), obtain,

$$
\varepsilon_{\alpha}^{*}=\frac{\alpha_{3}\left(\alpha_{4} \beta_{3}+\alpha_{2}\right)\left(\alpha_{4} \beta_{2}+\alpha_{1}\right)-\alpha_{3} \alpha_{3} \alpha_{4} \beta_{1}}{\left(\alpha_{4} \beta_{2}+\alpha_{1}\right)^{2}}
$$

Sub-class 2: $\alpha_{4}, \alpha_{3}, \alpha_{2}, \alpha_{1}, \beta_{3}, \beta_{2}$ and $\beta_{1}$ are multiplied by $\varepsilon_{\alpha}^{-1}$, and then substituting them into (7), obtain,

$$
\alpha_{3}\left(\alpha_{4} \beta_{3}+\varepsilon_{\alpha} \alpha_{2}\right)\left(\alpha_{4} \beta_{2}+\varepsilon_{\alpha} \alpha_{1}\right)>\varepsilon_{\alpha}\left(\alpha_{4} \beta_{2}+\varepsilon_{\alpha} \alpha_{1}\right)^{2}+\varepsilon_{\alpha} \alpha_{3} \alpha_{3} \alpha_{4} \beta_{1}
$$

For this sub-class, although the situation is complex, a moderate solution can still be obtained, that is,

$$
\varepsilon_{\alpha}^{*}=\frac{\alpha_{3} \alpha_{4} \alpha_{4} \beta_{2} \beta_{3}}{\left(\alpha_{4} \beta_{2}+\alpha_{1}\right)^{2}+\alpha_{3} \alpha_{3} \alpha_{4} \beta_{1}}
$$

From the demonstration above, it is obvious that for $n=2, n=3$ and $\varepsilon_{\beta}=1$ or $\varepsilon_{\beta}=\varepsilon_{\alpha}$ of the system matrix $A$, there all exist $\varepsilon_{\alpha}^{*}$ such that the system matrix $A$ is Hurwitz for all $0<\varepsilon_{\alpha}<\varepsilon_{\alpha}^{*}$. Moreover, Hurwitz stability condition is more and more complex as the order of the system matrix $A$ increases. Therefore, for the high order system matrix $A$, the same result can be still obtained with the help of computer. Thus, we can conclude that the $n+1$-order system matrix $A$ can be designed to be Hurwitz for all $0<\varepsilon_{\alpha}<\varepsilon_{\alpha}^{*}$ and $0<\varepsilon_{\beta}<\varepsilon_{\beta}^{*}$. As a result, the following theorem can be established.

Theorem 1: There exist $\alpha_{n+1}, \alpha_{n}, \cdots, \alpha_{2}, \alpha_{1}>0$ and $\beta_{n}, \beta_{n-1}, \cdots, \beta_{2}, \beta_{1}>0$ such that the system matrix $A$ for $\varepsilon_{\alpha}=\varepsilon_{\beta}=1$ is Hurwitz, and then it is still Hurwitz for all $0<\varepsilon_{\alpha}<\varepsilon_{\alpha}^{*}$ and $0<\varepsilon_{\beta}<\varepsilon_{\beta}^{*}$.

Discussion 1: From the system matrix $A$, the polynomial (2) and Hurwitz stability condition (3), it is not hard to see that: 1 ) when $\beta_{2}=0$, the control law is reduced to PID control; 2) for $\beta_{2}>0$, if $\alpha_{1}$ is less than zero, and then Hurwitz stability condition can still be satisfied. However, for PID control, $\alpha_{1}>0$ is the necessary condition. This means that linear general integral control is more robust than PID control.

Discussion 2: From the polynomial (1), it is not hard to see that that Hurwitz stability of the system matrix $A$ can still be achieved for $\alpha_{n+1}, \alpha_{n}, \cdots, \alpha_{2}, \alpha_{1}>0$ and $\beta_{n}, \cdots, \beta_{2} \leq 0$, or $\alpha_{n-1}, \cdots, \alpha_{1} \leq 0$ and $\beta_{n}, \cdots, \beta_{1}>0$. Therefore, the stability condition of Theorem 1 can be relaxed, but this is useless for the control design.

Discussion 3: From the statements above, Hurwitz stability condition is more and more complex as the order of the system matrix $A$ increases. So, although Theorem 1 is demonstrated by the single variable system matrix $A$, it is easy to extend Theorem 1 to the multiple variable case since there is not any difficulty to ensure that as any row controller gains, or controller and its integrator gains increase with the same ratio, the system matrix $A$ is always Hurwitz in theory, that is, Routh's stability criterion applies to not only the single variable system matrix but also the multiple variable one. Thus, the following proposition can be concluded.

Proposition 1: A canonical system matrix can be designed to be always Hurwitz as any row controller gains, or controller and its integrator gains increase with the same ratio.

\subsection{Solution of Lyapunov Equation}

By Hurwitz stability condition given by Subsection 2.1, the system matrix $A$ can be ensured to be Hurwitz for all $0<\varepsilon_{\alpha}<\varepsilon_{\alpha}^{*}$ and $0<\varepsilon_{\beta}<\varepsilon_{\beta}^{*}$. Thus, by linear system theory, if the system matrix $A$ is Hurwitz, and then for any given positive define symmetric matrix $Q$ there exists a unique positive define symmetric matrix $P$ that satisfies Lyapunov equation $P A+A^{\mathrm{T}} P=-Q$. Thus, the solution of Lyapunov equation can be obtained by skew symmetric matrix approach [10], that is,

$$
P=0.5(S-Q) A^{-1}
$$


where

$$
S=P A-A^{\mathrm{T}} P, S^{\mathrm{T}}=-S \text { and } A^{\mathrm{T}} S+S A=A^{\mathrm{T}} Q-Q A
$$

The inversion of the system matrix $A$ with $\varepsilon_{\alpha}=\varepsilon_{\beta}=1$ is,

$$
A^{-1}=\left[\begin{array}{cccccc}
* & * & \cdots & * & 0 & * \\
1 & 0 & \cdots & 0 & 0 & 0 \\
0 & 1 & \cdots & 0 & 0 & 0 \\
0 & 0 & \ddots & 0 & \vdots & \vdots \\
0 & 0 & \cdots & 1 & 0 & 0 \\
* & * & \cdots & * & -\alpha_{n+1}^{-1} & *
\end{array}\right]
$$

where the elements $*$ are omitted since it is useless to achieve our object. The interesting reader can evaluate them by $A A^{-1}=I$.

It is well known that the solution $P$ of Lyapunov equation is more and more complex as the order of the system matrix $A$ increases. Therefore, for clearly showing the results, we consider a simple case, that is, taking $Q=I$ and $n=2$ of the system matrix $A$. Thus, taking $\varepsilon_{\alpha}=\varepsilon_{\beta}=1$, obtain,

$$
S-Q=\left[\begin{array}{ccc}
-1 & S_{12} & S_{13} \\
-S_{12} & -1 & S_{23} \\
-S_{13} & -S_{23} & -1
\end{array}\right] \text { and } A^{-1}=\left[\begin{array}{ccc}
* & 0 & * \\
1 & 0 & 0 \\
* & -\left(\alpha_{3}\right)^{-1} & *
\end{array}\right]
$$

where

$$
\begin{aligned}
& s_{12}=\frac{\alpha_{1}+\left(\alpha_{1}+\alpha_{3} \beta_{2}\right) \alpha_{1}+\beta_{1} \beta_{1}+\alpha_{1} \beta_{2} \beta_{2}-\alpha_{2} \beta_{1} \beta_{2}}{\alpha_{2}\left(\alpha_{3} \beta_{2}+\alpha_{1}\right)-\alpha_{3} \beta_{1}}, \\
& s_{13}=\frac{\alpha_{1} \alpha_{2} \beta_{2}-\alpha_{3} \beta_{2} \beta_{1}-\alpha_{2} \alpha_{2} \beta_{1}-\alpha_{2} \alpha_{3}-\alpha_{3} \alpha_{3} \beta_{1}}{\alpha_{2}\left(\alpha_{3} \beta_{2}+\alpha_{1}\right)-\alpha_{3} \beta_{1}},
\end{aligned}
$$

and

$$
s_{23}=-\frac{\alpha_{3}+\alpha_{3}\left(\alpha_{3} \beta_{2}+\alpha_{1}\right)+\alpha_{3} \beta_{2} \beta_{2}+\alpha_{2} \beta_{1}}{\alpha_{2}\left(\alpha_{3} \beta_{2}+\alpha_{1}\right)-\alpha_{3} \beta_{1}} .
$$

and then we have,

$$
p_{21}=-\frac{s_{13}}{2 \alpha_{3}}, \quad p_{22}=-\frac{s_{23}}{2 \alpha_{3}}, \text { and } p_{23}=\frac{1}{2 \alpha_{3}}
$$

Case 1: $\alpha_{3}, \alpha_{2}$ and $\alpha_{1}$ are multiplied by $\varepsilon_{\alpha}^{-1}$, and then we have,

$$
\begin{aligned}
& s_{13}^{\varepsilon \alpha}=\frac{\alpha_{1} \alpha_{2} \beta_{2}-\varepsilon_{\alpha} \alpha_{3} \beta_{2} \beta_{1}-\alpha_{2} \alpha_{2} \beta_{1}-\alpha_{2} \alpha_{3}-\alpha_{3} \alpha_{3} \beta_{1}}{\alpha_{2}\left(\alpha_{3} \beta_{2}+\alpha_{1}\right)-\varepsilon_{\alpha} \alpha_{3} \beta_{1}}, \\
& s_{23}^{\varepsilon \alpha}=-\frac{\varepsilon_{\alpha} \alpha_{3}+\alpha_{3} \alpha_{3} \beta_{2}+\alpha_{1} \alpha_{3}+\varepsilon_{\alpha} \alpha_{3} \beta_{2} \beta_{2}+\varepsilon_{\alpha} \alpha_{2} \beta_{1}}{\alpha_{2}\left(\alpha_{3} \beta_{2}+\alpha_{1}\right)-\varepsilon_{\alpha} \alpha_{3} \beta_{1}}, \\
& p_{21}=-\frac{s_{13}^{\varepsilon \alpha}}{2 \alpha_{3}} \varepsilon_{\alpha}, \quad p_{22}=-\frac{s_{23}^{\varepsilon \alpha}}{2 \alpha_{3}} \varepsilon_{\alpha}, \text { and } p_{23}=\frac{1}{2 \alpha_{3}} \varepsilon_{\alpha} .
\end{aligned}
$$

It is obvious that $s_{13}^{\varepsilon \alpha}$ and $s_{23}^{\varepsilon \alpha}$ all tend to the constants as $\varepsilon_{\alpha} \rightarrow 0$, and then we have,

$$
\left\|P_{2}^{\alpha}\right\|=\left\|P_{2}^{\varepsilon \alpha}\right\| \varepsilon_{\alpha} \rightarrow 0 \text { as } \varepsilon_{\alpha} \rightarrow 0
$$

where $P_{2}^{\alpha}=P_{2}^{\varepsilon \alpha} \varepsilon_{\alpha}=\left[\begin{array}{lll}p_{21} & p_{22} & p_{23}\end{array}\right]$ and $P_{2}^{\varepsilon \alpha}=-0.5 \alpha_{3}^{-1}\left[\begin{array}{ccc}s_{13}^{\varepsilon \alpha} & s_{23}^{\varepsilon \alpha} & -1\end{array}\right]$. 
Case 2: $\alpha_{3}, \alpha_{2}, \alpha_{1}, \beta_{2}$ and $\beta_{1}$ are multiplied by $\varepsilon_{\alpha}^{-1}$, and then we have,

$$
\begin{aligned}
& s_{13}^{\varepsilon \alpha \beta}=\frac{\alpha_{1} \alpha_{2} \beta_{2}-\alpha_{3} \beta_{2} \beta_{1}-\alpha_{2} \alpha_{2} \beta_{1}-\varepsilon_{\alpha} \alpha_{2} \alpha_{3}-\alpha_{3} \alpha_{3} \beta_{1}}{\alpha_{2}\left(\alpha_{3} \beta_{2}+\varepsilon_{\alpha} \alpha_{1}\right)-\varepsilon_{\alpha} \alpha_{3} \beta_{1}}, \\
& s_{23}^{\varepsilon \alpha \beta}=-\frac{\varepsilon_{\alpha} \varepsilon_{\alpha} \alpha_{3}+\alpha_{3} \alpha_{3} \beta_{2}+\varepsilon_{\alpha} \alpha_{1} \alpha_{3}+\alpha_{3} \beta_{2} \beta_{2}+\varepsilon_{\alpha} \alpha_{2} \beta_{1}}{\alpha_{2}\left(\alpha_{3} \beta_{2}+\varepsilon_{\alpha} \alpha_{1}\right)-\varepsilon_{\alpha} \alpha_{3} \beta_{1}}, \\
& p_{21}=-\frac{s_{13}^{\varepsilon \alpha \beta}}{2 \alpha_{3}} \varepsilon_{\alpha}, \quad p_{22}=-\frac{s_{23}^{\varepsilon \alpha \beta}}{2 \alpha_{3}} \varepsilon_{\alpha}, \text { and } p_{23}=\frac{1}{2 \alpha_{3}} \varepsilon_{\alpha} .
\end{aligned}
$$

It is obvious that $S_{13}^{\varepsilon \alpha \beta}$ and $s_{23}^{\varepsilon \alpha \beta}$ all tend to the constants as $\varepsilon_{\alpha} \rightarrow 0$, and then we have,

$$
\left\|P_{2}^{\alpha \beta}\right\|=\left\|P_{2}^{\varepsilon \alpha \beta}\right\| \varepsilon_{\alpha} \rightarrow 0 \text { and } \varepsilon_{\alpha} \rightarrow 0
$$

where $P_{2}^{\alpha \beta}=P_{2}^{\varepsilon \alpha \beta} \varepsilon_{\alpha}=\left[\begin{array}{lll}p_{21} & p_{22} & p_{23}\end{array}\right]$ and $P_{2}^{\varepsilon \alpha \beta}=-0.5 \alpha_{3}^{-1}\left[\begin{array}{lll}s_{13}^{\varepsilon \alpha \beta} & s_{23}^{\varepsilon \alpha \beta} & -1\end{array}\right]$.

From the statements above, it is easy to see that for $n=2$ and $\varepsilon_{\beta}=1$ or $\varepsilon_{\beta}=\varepsilon_{\alpha}$ of the system matrix $A$, $\left\|P_{2}^{\alpha}\right\|$ and $\left\|P_{2}^{\alpha \beta}\right\|$ can all be formulated as the linear form on $\varepsilon_{\alpha}$ and all tend to zero as $\varepsilon_{\alpha} \rightarrow 0$. Moreover, the solution of the matrix $S$ is more and more complex as the order of the system matrix $A$ increases. Thus, by the inversion matrix $A^{-1}$ (9), $\left\|P_{n}^{\alpha}\right\|$ and $\left\|P_{n}^{\alpha \beta}\right\|$ can all be formulated as the linear form on $\varepsilon_{\alpha}$ for the $n+1$-order system matrix $A$, and with the help of computer, it can be verified that the solutions of $P_{n}^{\varepsilon \alpha}$ and $P_{n}^{\varepsilon \alpha \beta}$ still tend to the constants as $\varepsilon_{\alpha} \rightarrow 0$. Therefore, for the $n+1$-order system matrix $A$, we can conclude that $\left\|P_{n}^{\alpha}\right\| \rightarrow 0$ as $\varepsilon_{\alpha} \rightarrow 0$ for all $0<\varepsilon_{\beta}<\varepsilon_{\beta}^{*}$, and $\left\|P_{n}^{\alpha \beta}\right\| \rightarrow 0$ as $\varepsilon_{\beta}=\varepsilon_{\alpha} \rightarrow 0$. As a result, the following theorem can be established.

Theorem 2: If there exist the gains $\alpha_{n+1}, \cdots, \alpha_{1}>0$ and $\beta_{n}, \cdots, \beta_{1}>0$ such that the $n+1$-order system matrix $A$ is Hurwitz for all $0<\varepsilon_{\alpha}<\varepsilon_{\alpha}^{*}$ and $0<\varepsilon_{\beta}<\varepsilon_{\beta}^{*}$, and then we have,

1) $\left\|P_{n}^{\alpha}\right\|=\left\|P_{n}^{\varepsilon \alpha}\right\| \varepsilon_{\alpha} \rightarrow 0 \quad \forall \varepsilon_{\beta} \in\left(0, \varepsilon_{\beta}^{*}\right)$ as $\varepsilon_{\alpha} \rightarrow 0$.

2) $\left\|P_{n}^{\alpha \beta}\right\|=\left\|P_{n}^{\varepsilon \alpha \beta}\right\| \varepsilon_{\alpha} \rightarrow 0$ as $\varepsilon_{\beta}=\varepsilon_{\alpha} \rightarrow 0$.

where

$$
\begin{aligned}
& P_{n}^{\alpha}=P_{n}^{\varepsilon \alpha} \varepsilon_{\alpha}=\left[\begin{array}{lll}
p_{n 1} & \cdots & p_{n, n+1}
\end{array}\right], \\
& P_{n}^{\varepsilon \alpha}=-0.5 \alpha_{n+1}^{-1}\left[\begin{array}{lllll}
s_{1, n+1}^{\varepsilon \alpha} & s_{2, n+1}^{\varepsilon \alpha} & \cdots & s_{n, n+1}^{\varepsilon \alpha} & -1
\end{array}\right], \\
& P_{n}^{\alpha \beta}=P_{n}^{\varepsilon \alpha \beta} \varepsilon_{\alpha}=\left[\begin{array}{lll}
p_{n 1} & \cdots & p_{n, n+1}
\end{array}\right], \\
& \text { and } \\
& P_{n}^{\varepsilon \alpha \beta}=-0.5 \alpha_{n+1}^{-1}\left[\begin{array}{lllll}
S_{1, n+1}^{\varepsilon \alpha \beta} & s_{2, n+1}^{\varepsilon \alpha \beta} & \cdots & s_{n, n+1}^{\varepsilon \alpha \beta} & -1
\end{array}\right] .
\end{aligned}
$$

Discussion 4: Theorem 1 and 2 are all obtained by multiplying the controller gains $\alpha_{i}$ and the integrator gains $\beta_{j}$ by the same ratios $\varepsilon_{\alpha}^{-1}$ and $\varepsilon_{\beta}^{-1}$, respectively, even under the typical cases $\varepsilon_{\beta}^{-1}$ can be equal to 1 or $\varepsilon_{\alpha}^{-1}$. This is the reason why our method is called as Equal ratio gain technique.

Discussion 5: From the statements above, the solution of the matrix $S$ is more and more complex as the order of the system matrix $A$ increases. So, although Theorem 2 is demonstrated by taking $Q=I$ and the single variable system matrix $A$, it is very easy to extend Theorem 2 to any given positive define symmetric matrix $Q$ and the multiple variable system matrix $A$ with the help of computer since there is not any difficulty to obtain the solution of the matrix $S$ in theory, that is, Lyapunov equation applies to not only the single system matrix but also the multiple system matrix. Thus, there is the following proposition.

Proposition 2: As any row controller gains, or controller and its integrator gains of a canonical system matrix tend to infinity with the same ratio, if it is always Hurwitz, and then the same row solutions of Lyapunov equation all tend to zero.

\subsection{Example}

For testifying the justification of Theorems 1 and 2, and Propositions 1 and 2, we consider a 6-order two varia- 
ble system matrix $A$ as follows,

$$
A=\left[\begin{array}{cccccc}
0 & 0 & 0 & 0 & 1 & 0 \\
0 & 0 & 0 & 0 & 0 & 1 \\
\beta_{1}^{x} & \beta_{2}^{x} & 0 & 0 & \beta_{3}^{x} & \beta_{4}^{x} \\
\beta_{1}^{y} & \beta_{2}^{y} & 0 & 0 & \beta_{3}^{y} & \beta_{4}^{y} \\
-\alpha_{1}^{x} & -\alpha_{2}^{x} & -\alpha_{3}^{x} & 0 & -\alpha_{4}^{x} & -\alpha_{5}^{x} \\
-\alpha_{1}^{y} & -\alpha_{2}^{y} & 0 & -\alpha_{3}^{y} & -\alpha_{4}^{y} & -\alpha_{5}^{y}
\end{array}\right]
$$

The polynomial of the system matrix $A$ is,

$$
a_{0} s^{6}+a_{1} s^{5}+a_{2} s^{4}+a_{3} s^{3}+a_{4} s^{2}+a_{5} s+a_{6}=0 .
$$

where

$$
\begin{aligned}
& a_{0}=1, a_{1}=\alpha_{4}^{x}+\alpha_{5}^{y}, a_{6}=\left(\beta_{1}^{x} \beta_{2}^{y}-\beta_{1}^{y} \beta_{2}^{x}\right) \alpha_{3}^{x} \alpha_{3}^{y}, a_{2}=\alpha_{4}^{x} \alpha_{5}^{y}-\alpha_{5}^{x} \alpha_{4}^{y}+\beta_{4}^{y} \alpha_{3}^{y}+\beta_{3}^{x} \alpha_{3}^{x}+\alpha_{2}^{y}+\alpha_{1}^{x}, \\
& a_{3}=\beta_{4}^{y} \alpha_{3}^{y} \alpha_{4}^{x}+\beta_{3}^{x} \alpha_{3}^{x} \alpha_{5}^{y}-\beta_{4}^{x} \alpha_{3}^{x} \alpha_{4}^{y}-\beta_{3}^{y} \alpha_{5}^{x} \alpha_{3}^{y}+\alpha_{2}^{y} \alpha_{4}^{x}-\alpha_{2}^{x} \alpha_{4}^{y}+\alpha_{3}^{y} \beta_{2}^{y}+\beta_{1}^{x} \alpha_{3}^{x}-\alpha_{5}^{x} \alpha_{1}^{y}+\alpha_{1}^{x} \alpha_{5}^{y}, \\
& a_{4}=\left(\beta_{3}^{x} \beta_{4}^{y}-\beta_{4}^{x} \beta_{3}^{y}\right) \alpha_{3}^{x} \alpha_{3}^{y}+\alpha_{1}^{x} \alpha_{2}^{y}-\alpha_{2}^{x} \alpha_{1}^{y}-\beta_{2}^{x} \alpha_{3}^{x} \alpha_{4}^{y}+\beta_{3}^{x} \alpha_{3}^{x} \alpha_{2}^{y}+\beta_{2}^{y} \alpha_{3}^{y} \alpha_{4}^{x}-\beta_{3}^{y} \alpha_{2}^{x} \alpha_{3}^{y}-\beta_{4}^{x} \alpha_{3}^{x} \alpha_{1}^{y} \\
& \quad+\beta_{1}^{x} \alpha_{3}^{x} \alpha_{5}^{y}-\beta_{1}^{y} \alpha_{5}^{x} \alpha_{3}^{y}+\beta_{4}^{y} \alpha_{1}^{x} \alpha_{3}^{y},
\end{aligned}
$$

and

$$
a_{5}=\left(\beta_{1}^{x} \beta_{4}^{y}-\beta_{4}^{x} \beta_{1}^{y}\right) \alpha_{3}^{x} \alpha_{3}^{y}-\left(\beta_{2}^{x} \beta_{3}^{y}-\beta_{3}^{x} \beta_{2}^{y}\right) \alpha_{3}^{x} \alpha_{3}^{y}-\beta_{2}^{x} \alpha_{3}^{x} \alpha_{1}^{y}+\beta_{1}^{x} \alpha_{3}^{x} \alpha_{2}^{y}-\beta_{1}^{y} \alpha_{2}^{x} \alpha_{3}^{y}+\beta_{2}^{y} \alpha_{1}^{x} \alpha_{3}^{y} .
$$

The inversion of the system matrix $A$ is,

$$
A^{-1}=\left[\begin{array}{cccccc}
* & * & * & * & 0 & 0 \\
* & * & * & * & 0 & 0 \\
* & * & * & * & -\left(\alpha_{3}^{x}\right)^{-1} & 0 \\
* & * & * & * & 0 & -\left(\alpha_{3}^{y}\right)^{-1} \\
1 & 0 & 0 & 0 & 0 & 0 \\
0 & 1 & 0 & 0 & 0 & 0
\end{array}\right]
$$

By the equation $A^{\mathrm{T}} S+S A=A^{\mathrm{T}} Q-Q A$, it is very easy to obtain the fifteen linear equations with fifteen elements of the matrix $S$. So, it is omitted.

Thus, taking

$$
A=\left[\begin{array}{cccccc}
0 & 0 & 0 & 0 & 1 & 0 \\
0 & 0 & 0 & 0 & 0 & 1 \\
8 & 2 & 0 & 0 & 3 & 1 \\
2 & 7 & 0 & 0 & 1 & 5 \\
-8 & -2 & -8 & 0 & -3 & -1 \\
-2 & -8 & 0 & -8 & -1 & -3
\end{array}\right] \text { and } Q=\left[\begin{array}{cccccc}
3.0 & 1.0 & -0.8 & 0.6 & 0.5 & 0.3 \\
1.0 & 5.0 & 1.3 & 1.0 & 0.8 & 0.2 \\
-0.8 & 1.3 & 6 & 0.8 & 0.4 & 1.0 \\
0.6 & 1.0 & 0.8 & 2.0 & 1.3 & 0.5 \\
0.5 & 0.8 & 0.4 & 1.3 & 3.0 & 0.8 \\
0.3 & 0.2 & 1.0 & 0.5 & 0.8 & 6.0
\end{array}\right] \text {, }
$$

and then by Routh's stability criterion, the array of the coefficients of polynomial is,

$$
\begin{array}{ccccc}
s^{6} & a_{0} & a_{2} & a_{4} & a_{6} \\
s^{5} & a_{1} & a_{3} & a_{5} & 0 \\
s^{4} & b_{1} & b_{2} & b_{3} & 0 \\
s^{3} & c_{1} & c_{2} & 0 & 0 \\
s^{2} & d_{1} & d_{2} & 0 & 0 \\
s^{1} & e_{1} & 0 & & \\
s^{0} & f_{1} & & &
\end{array}
$$


where

$$
\begin{aligned}
& a_{0}=1, \quad a_{1}=6, \quad a_{2}=88, a_{3}=340, \quad a_{4}=1764, \quad a_{5}=4544, \quad a_{6}=3328, \\
& b_{3}=a_{6}, \quad b_{1}=\frac{a_{1} a_{2}-a_{0} a_{3}}{a_{1}}, \quad b_{2}=\frac{a_{1} a_{4}-a_{0} a_{5}}{a_{1}}, \quad c_{1}=\frac{b_{1} a_{3}-a_{1} b_{2}}{b_{1}}, \quad c_{2}=\frac{b_{1} a_{5}-a_{1} b_{3}}{b_{1}}, \\
& d_{1}=\frac{c_{1} b_{2}-b_{1} c_{2}}{c_{1}}, d_{2}=\frac{c_{1} b_{3}-b_{1} c_{3}}{c_{1}}, e_{1}=\frac{d_{1} c_{2}-c_{1} d_{2}}{d_{1}}, \text { and } f_{1}=d_{2} .
\end{aligned}
$$

Now, with the help of computer, we have: 1) if $\alpha_{i}^{x} \quad(i=1,2, \cdots, 5)$ of the system matrix $A$ are multiplied by $\varepsilon^{-1}$, and then it is still Hurwitz for all $\left.0<\varepsilon \leq \varepsilon^{*}=1.25 ; 2\right)$ if $\alpha_{i}^{x}$ and $\beta_{j}^{x}(j=1,2, \cdots, 4)$ of the system matrix $A$ are multiplied by $\varepsilon^{-1}$, and then it is still Hurwitz for all $0<\varepsilon \leq \varepsilon^{*}=1.45$; 3) if $\alpha_{i}^{y}$ of the system matrix $A$ are multiplied by $\varepsilon^{-1}$, and then it is still Hurwitz for all $0<\varepsilon \leq \varepsilon^{*}=1.72$;4) if $\alpha_{i}^{y}$ and $\beta_{j}^{y}$ of the system matrix $A$ are multiplied by $\varepsilon^{-1}$, then it is still Hurwitz for all $0<\varepsilon \leq \varepsilon^{*}=2.90$; 5) if $\alpha_{i}^{x}$ and $\alpha_{i}^{y}$ of the system matrix $A$ are multiplied by $\varepsilon^{-1}$, and then it is still Hurwitz for all $0<\varepsilon \leq \varepsilon^{*}=1.2$, and the numerical solutions of $P_{5}$ and $P_{6}$ are shown in Table 1 and Table 2, respectively; 6) if $\alpha_{i}^{x}, \alpha_{i}^{y}, \beta_{j}^{x}$ and $\beta_{j}^{y}$ of the system matrix $A$ are multiplied by $\varepsilon^{-1}$, and then it is still Hurwitz for all $0<\varepsilon \leq \varepsilon^{*}=1.28$, and the numerical solutions of $P_{5}$ and $P_{6}$ are shown in Table 3 and Table 4, respectively.

Table 1. Numerical Solutions of $P_{5}$ for all $\alpha_{i}^{x}$ and $\alpha_{i}^{y}$ multiplied by $\varepsilon^{-1}$.

\begin{tabular}{cccc}
\hline & $\varepsilon=1.0$ & $\varepsilon=0.1$ & $\varepsilon=0.01$ \\
\hline$p_{51}$ & 22.43 & $2.72 \mathrm{e}-1$ & $2.34 \mathrm{e}-2$ \\
$p_{52}$ & -2.24 & $6.53 \mathrm{e}-3$ & $3.99 \mathrm{e}-4$ \\
$p_{53}$ & -0.75 & $7.50 \mathrm{e}-2$ & $7.50 \mathrm{e}-3$ \\
$p_{54}$ & 0.94 & $7.66 \mathrm{e}-2$ & $7.40 \mathrm{e}-3$ \\
$p_{55}$ & 10.86 & $1.34 \mathrm{e}-1$ & $1.16 \mathrm{e}-2$ \\
$p_{56}$ & -3.95 & $-4.43 \mathrm{e}-2$ & $-4.15 \mathrm{e}-3$ \\
\hline
\end{tabular}

Table 2. Numerical Solutions of $P_{6}$ for all $\alpha_{i}^{x}$ and $\alpha_{i}^{y}$ multiplied by $\varepsilon^{-1}$.

\begin{tabular}{cccc}
\hline & $\varepsilon=1.0$ & $\varepsilon=0.1$ & $\varepsilon=0.01$ \\
\hline$p_{61}$ & -9.42 & $-1.59 \mathrm{e}-1$ & $-1.49 \mathrm{e}-2$ \\
$p_{62}$ & 5.20 & $2.46 \mathrm{e}-1$ & $2.36 \mathrm{e}-2$ \\
$p_{63}$ & -0.84 & $-6.66 \mathrm{e}-2$ & $-6.40 \mathrm{e}-3$ \\
$p_{64}$ & 0.25 & $2.50 \mathrm{e}-2$ & $2.50 \mathrm{e}-3$ \\
$p_{65}$ & -3.95 & $-4.43 \mathrm{e}-2$ & $-4.15 \mathrm{e}-3$ \\
$p_{66}$ & 5.19 & $2.25 \mathrm{e}-1$ & $2.15 \mathrm{e}-2$ \\
\hline
\end{tabular}

Table 3. Numerical Solutions of $P_{5}$ for all $\alpha_{i}^{x}, \alpha_{i}^{y}, \beta_{j}^{x}$ and $\beta_{j}^{y}$ multiplied by $\varepsilon^{-1}$.

\begin{tabular}{lccl}
\hline & $\varepsilon=1.0$ & $\varepsilon=0.1$ & $\varepsilon=0.01$ \\
\hline$p_{51}$ & 22.43 & $2.74 \mathrm{e}-1$ & $2.39 \mathrm{e}-2$ \\
$p_{52}$ & -2.24 & $9.96 \mathrm{e}-2$ & $9.55 \mathrm{e}-3$ \\
$p_{53}$ & -0.75 & $7.50 \mathrm{e}-2$ & $7.50 \mathrm{e}-3$ \\
$p_{54}$ & 0.94 & $8.98 \mathrm{e}-2$ & $8.57 \mathrm{e}-3$ \\
$p_{55}$ & 10.86 & $1.37 \mathrm{e}-1$ & $1.20 \mathrm{e}-2$ \\
$p_{56}$ & -3.95 & $2.85 \mathrm{e}-2$ & $3.01 \mathrm{e}-3$ \\
\hline
\end{tabular}


Table 4. Numerical Solutions of $P_{6}$ for all $\alpha_{i}^{x}, \alpha_{i}^{y}, \beta_{j}^{x}$ and $\beta_{j}^{y}$ multiplied by $\varepsilon^{-1}$.

\begin{tabular}{cccc}
\hline & $\varepsilon=1.0$ & $\varepsilon=0.1$ & $\varepsilon=0.01$ \\
\hline$p_{61}$ & -9.42 & $4.26 \mathrm{e}-3$ & $1.33 \mathrm{e}-3$ \\
$p_{62}$ & 5.20 & $3.15 \mathrm{e}-1$ & $3.10 \mathrm{e}-2$ \\
$p_{63}$ & -0.84 & $-7.98 \mathrm{e}-2$ & $-7.57 \mathrm{e}-3$ \\
$p_{64}$ & 0.25 & $2.50 \mathrm{e}-2$ & $2.5 \mathrm{e}-3$ \\
$p_{65}$ & -3.95 & $2.85 \mathrm{e}-2$ & $3.01 \mathrm{e}-3$ \\
$p_{66}$ & 5.19 & $2.40 \mathrm{e}-1$ & $2.32 \mathrm{e}-2$ \\
\hline
\end{tabular}

From the example above, it is obvious that: 1) for all the six cases, there all exists $\varepsilon^{*}$ such that the system matrix $A$ is always Hurwitz for all $0<\varepsilon \leq \varepsilon^{*} ; 2$ ) as shown in Tables 1 -4, the absolute values of $p_{5 i}$ and $p_{6 i}(i=1,2, \cdots, 6)$ are all decrease as $\varepsilon$ reduces. This not only verifies the results proposed by Subsection 2.1 and 2.2 but also shows that for the high order and multiple variable system matrix $A$, it is convenient and practical with the help of computer.

\section{Control Design}

Consider the following controllable nonlinear system,

$$
\left\{\begin{array}{l}
\dot{x}_{1}=x_{2} \\
\dot{x}_{2}=x_{3} \\
\quad \vdots \\
\dot{x}_{n}=f_{x}(x, y, w)+g_{x}(x, y, w) u_{x} \\
\dot{y}_{1}=y_{2} \\
\dot{y}_{2}=y_{3} \\
\quad \vdots \\
\dot{y}_{m}=f_{y}(x, y, w)+g_{y}(x, y, w) u_{y}
\end{array}\right.
$$

where $x \in R^{n}$ and $y \in R^{m}$ are the states; $u_{x}, u_{y} \in R$ are the control inputs; $w \in R^{l}$ is a vector of unknown constant parameters and disturbances. The functions $f_{x}(x, y, w)$ and $f_{y}(x, y, w)$ are uncertain nonlinear actions, and the functions $g_{x}(x, y, w)$ and $g_{y}(x, y, w)$ are continuous in $(x, y, w)$ on the control domain $D_{x} \times D_{y} \times D_{w} \subset R^{n} \times R^{m} \times R^{l}$. We want to design the control laws $u_{x}$ and $u_{y}$ such that $x(t), y(t) \rightarrow 0$ as $t \rightarrow \infty$.

Assumption 1: There are two unique control inputs $u_{x 0}$ and $u_{y 0}$ that satisfy the equations,

$$
\begin{aligned}
& 0=f_{x}(0,0, w)+g_{x}(0,0, w) u_{x 0} \\
& 0=f_{y}(0,0, w)+g_{y}(0,0, w) u_{y 0}
\end{aligned}
$$

so that $x=y=0$ is the desired equilibrium point, and $u_{x 0}$ and $u_{y 0}$ are the steady-state controls that are needed to maintain equilibrium at $x=y=0$.

Assumption 2: No loss of generality, suppose that the functions $f_{x}(x, y, w), f_{y}(x, y, w), g_{x}(x, y, w)$ and $g_{y}(x, y, w)$ satisfy the following inequalities,

$$
\begin{gathered}
g_{x}(x, y, w)>g_{m}^{x}>0 \\
g_{y}(x, y, w)>g_{m}^{y}>0 \\
\left\|f_{x}(x, y, w)-f_{x}(0,0, w)\right\| \leq l_{f_{x}}^{x}\|x\|+l_{f_{x}}^{y}\|y\|
\end{gathered}
$$




$$
\begin{gathered}
\left\|f_{y}(x, y, w)-f_{y}(0,0, w)\right\| \leq l_{f_{y}}^{x}\|x\|+l_{f_{y}}^{y}\|y\| \\
\left\|g_{x}(x, y, w)-g_{x}(0,0, w)\right\| \leq l_{g_{x}}^{x}\|x\|+l_{g_{x}}^{y}\|y\| \\
\left\|g_{y}(x, y, w)-g_{y}(0,0, w)\right\| \leq l_{g y}^{x}\|x\|+l_{g_{y}}^{y}\|y\| \\
\left\|f_{x}(0,0, w) g_{x}^{-1}(0,0, w)\right\| \leq \gamma_{x} \\
\left\|f_{y}(0,0, w) g_{y}^{-1}(0,0, w)\right\| \leq \gamma_{y}
\end{gathered}
$$

for all $x \in D_{x}, \quad y \in D_{y}$ and $w \in D_{w}$. where $l_{f_{x}}^{x}, l_{f_{x}}^{y}, l_{f_{y}}^{x}, l_{f_{y}}^{y}, l_{g_{x}}^{x}, l_{g_{x}}^{y}, l_{g_{y}}^{x}, l_{g_{y}}^{y}, \gamma_{x}, \gamma_{y}, g_{m}^{x}$ and $g_{m}^{y}$ are all positive constants.

For the system (10), we develop two kinds of methods to design linear general integral controllers, respectively, that is, Decomposition method and Synthetic method.

\subsection{Decomposition Method}

The control laws $u_{x}$ and $u_{y}$ are taken as,

$$
\begin{aligned}
& \left\{\begin{array}{l}
u_{x}=-\varepsilon_{\alpha x}^{-1}\left(\alpha_{1}^{x} x_{1}+\alpha_{2}^{x} x_{2}+\cdots+\alpha_{n}^{x} x_{n}+\alpha_{n+1}^{x} \sigma_{x}\right) \\
\dot{\sigma}_{x}=\varepsilon_{\beta x}^{-1}\left(\beta_{1}^{x} x_{1}+\beta_{2}^{x} x_{2}+\cdots+\beta_{n}^{x} x_{n}\right)
\end{array}\right. \\
& \left\{\begin{array}{l}
u_{y}=-\varepsilon_{\alpha y}^{-1}\left(\alpha_{1}^{y} y_{1}+\alpha_{2}^{y} y_{2}+\cdots+\alpha_{m}^{y} y_{m}+\alpha_{m+1}^{y} \sigma_{y}\right) \\
\dot{\sigma}_{y}=\varepsilon_{\beta y}^{-1}\left(\beta_{1}^{y} y_{1}+\beta_{2}^{y} y_{2}+\cdots+\beta_{m}^{y} y_{m}\right)
\end{array}\right.
\end{aligned}
$$

where $\alpha_{i}^{x}, \beta_{j}^{x}, \alpha_{k}^{y}, \beta_{l}^{y}, \varepsilon_{\alpha x}, \varepsilon_{\alpha y}, \varepsilon_{\beta x}$ and $\varepsilon_{\beta y}$ are all positive constants $(i=1,2, \cdots n+1, j=1,2, \cdots n$, $k=1,2, \cdots m+1$ and $l=1,2, \cdots m)$.

Thus, substituting (21) and (22) into (10), obtain two augmented systems,

$$
\begin{aligned}
& \left\{\begin{array}{l}
\dot{x}_{1}=x_{2} \\
\dot{x}_{2}=x_{3} \\
\quad \vdots \\
\dot{x}_{n}=f_{x}(x, y, w)-g_{x}(x, y, w) \varepsilon_{\alpha x}^{-1}\left(\alpha_{1}^{x} x_{1}+\alpha_{2}^{x} x_{2}+\cdots+\alpha_{n}^{x} x_{n}+\alpha_{n+1}^{x} \sigma_{x}\right) \\
\dot{\sigma}_{x}=\varepsilon_{\beta x}^{-1}\left(\beta_{1}^{x} x_{1}+\beta_{2}^{x} x_{2}+\cdots+\beta_{n}^{x} x_{n}\right)
\end{array}\right. \\
& \left\{\begin{array}{l}
\dot{y}_{1}=y_{2} \\
\dot{y}_{2}=y_{3} \\
\quad \vdots \\
\dot{y}_{m}=f_{y}(x, y, w)-g_{y}(x, y, w) \varepsilon_{\alpha y}^{-1}\left(\alpha_{1}^{y} y_{1}+\alpha_{2}^{y} y_{2}+\cdots+\alpha_{m}^{y} y_{m}+\alpha_{m+1}^{y} \sigma_{y}\right) \\
\dot{\sigma}_{y}=\varepsilon_{\beta y}^{-1}\left(\beta_{1}^{y} y_{1}+\beta_{2}^{y} y_{2}+\cdots+\beta_{m}^{y} y_{m}\right)
\end{array}\right.
\end{aligned}
$$

By Assumption 1 and choosing $\varepsilon_{\alpha x}^{-1} \alpha_{n+1}^{x}$ to be large enough, and then setting $\dot{x}=0$ and $x=y=0$ of the system (23), we obtain,

$$
g_{x}(0,0, w) \varepsilon_{\alpha x}^{-1} \alpha_{n+1}^{x} \sigma_{x 0}=f_{x}(0,0, w)
$$

In the same way, we have,

$$
g_{y}(0,0, w) \varepsilon_{\alpha y}^{-1} \alpha_{m+1}^{y} \sigma_{y 0}=f_{y}(0,0, w)
$$


Thus, we ensure that there are two unique solutions $\sigma_{x 0}$ and $\sigma_{y 0}$, and then $\left(0,0, \sigma_{x 0}\right)$ and $\left(0,0, \sigma_{y 0}\right)$ are the unique equilibrium points of the systems (23) and (24), respectively.

Substituting (25) into (23) and (26) into (24), and then the whole closed-loop system can be rewritten as,

$$
\left\{\begin{array}{l}
\dot{\eta}_{x}=A_{x} \eta_{x}+F_{x}(x, y, w) \\
\dot{\eta}_{y}=A_{y} \eta_{y}+F_{y}(x, y, w)
\end{array}\right.
$$

where $\eta_{x}=\left[\begin{array}{ll}x^{\mathrm{T}} & \sigma_{x}-\sigma_{x 0}\end{array}\right]^{\mathrm{T}}, \quad \eta_{y}=\left[\begin{array}{ll}y^{\mathrm{T}} & \sigma_{y}-\sigma_{y 0}\end{array}\right]^{\mathrm{T}}$,

$$
\begin{aligned}
A_{x} & =\left[\begin{array}{ccccc}
0 & 1 & \cdots & 0 & 0 \\
0 & 0 & \ddots & 0 & 0 \\
0 & 0 & \cdots & 1 & 0 \\
-\varepsilon_{\alpha x}^{-1} \alpha_{1}^{x} & -\varepsilon_{\alpha x}^{-1} \alpha_{2}^{x} & \cdots & -\varepsilon_{\alpha x}^{-1} \alpha_{n}^{x} & -\varepsilon_{\alpha x}^{-1} \alpha_{n+1}^{x} \\
\varepsilon_{\beta x}^{-1} \beta_{1}^{x} & \varepsilon_{\beta x}^{-1} \beta_{2}^{x} & \cdots & \varepsilon_{\beta x}^{-1} \beta_{n}^{x} & 0
\end{array}\right], \\
A_{y} & =\left[\begin{array}{ccccc}
0 & 1 & \cdots & 0 & 0 \\
0 & 0 & \ddots & 0 & 0 \\
0 & 0 & \cdots & 1 & 0 \\
-\varepsilon_{\alpha x}^{-1} \alpha_{1}^{y} & -\varepsilon_{\alpha y}^{-1} \alpha_{2}^{y} & \cdots & -\varepsilon_{\alpha y}^{-1} \alpha_{m}^{y} & -\varepsilon_{\alpha y}^{-1} \alpha_{m+1}^{y} \\
\varepsilon_{\beta y}^{-1} \beta_{1}^{y} & \varepsilon_{\beta y}^{-1} \beta_{2}^{y} & \cdots & \varepsilon_{\beta y}^{-1} \beta_{m}^{y} & 0
\end{array}\right]
\end{aligned}
$$

$F_{x}(x, y, w)$ and $F_{y}(x, y, w)$ are $n+1 \times 1$ and $m+1 \times 1$ matrices, respectively, all their elements are equal to zero except for

$$
f_{n 1}^{x}=f_{x}(x, y, w)-f_{x}(0,0, w)-\left[g_{x}(x, y, w)-g_{x}(0,0, w)\right] f_{x}(0,0, w) g_{x}^{-1}(0,0, w)
$$

and

$$
f_{m 1}^{y}=f_{y}(x, y, w)-f_{y}(0,0, w)-\left[g_{y}(x, y, w)-g_{y}(0,0, w)\right] f_{y}(0,0, w) g_{y}^{-1}(0,0, w) .
$$

Moreover, it is worthy to note that the functions $g_{x}(x, y, w)$ and $g_{y}(x, y, w)$ are integrated into $\varepsilon_{\alpha x}$ and $\varepsilon_{\alpha y}$, respectively, via a change of variable. This has not influence on the results if the inequalities (13) and (14) hold and they can be taken as $g_{m}^{x}$ and $g_{m}^{y}$, respectively, in the design. Therefore, they are omitted in all the following demonstrations.

The matrices $A_{x}$ for all $0<\varepsilon_{\beta x}<\varepsilon_{\beta x}^{*}$ and $0<\varepsilon_{\alpha x}<\varepsilon_{\alpha x}^{*}$, and the matrix $A_{y}$ for all $0<\varepsilon_{\beta y}<\varepsilon_{\beta y}^{*}$ and $0<\varepsilon_{\alpha y}<\varepsilon_{\alpha y}^{*}$ can be designed to be Hurwitz, respectively. Thus, two quadratic Lyapunov functions,

$$
\begin{aligned}
& V_{x}\left(\eta_{x}\right)=\eta_{x}^{\mathrm{T}} P_{x} \eta_{x} \\
& V_{y}\left(\eta_{y}\right)=\eta_{y}^{\mathrm{T}} P_{y} \eta_{y}
\end{aligned}
$$

can be obtained. Where $P_{x}$ and $P_{y}$ are the solutions of Lyapunov equations

$$
P_{x} A_{x}+A_{x}^{\mathrm{T}} P_{x}=-Q_{x} \text { and } P_{y} A_{y}+A_{y}^{\mathrm{T}} P_{y}=-Q_{y}
$$

with any given positive define symmetric matrices $Q_{x}$ and $Q_{y}$, respectively.

Thus, using $V\left(\eta_{x}, \eta_{y}\right)=V_{x}\left(\eta_{x}\right)+V_{y}\left(\eta_{y}\right)$ as Lyapunov function candidate, and then its time derivative along the trajectories of the closed-loop system (27) is,

$$
\begin{aligned}
\dot{V}\left(\eta_{x}, \eta_{y}\right) & =\eta_{x}^{\mathrm{T}}\left(P_{x} A_{x}+A_{x}^{\mathrm{T}} P_{x}\right) \eta_{x}+\frac{\partial V_{x}\left(\eta_{x}\right)}{\partial \eta_{x}} F_{x}(x, y, w)+\eta_{y}^{\mathrm{T}}\left(P_{y} A_{y}+A_{y}^{\mathrm{T}} P_{y}\right) \eta_{y}+\frac{\partial V_{y}\left(\eta_{y}\right)}{\partial \eta_{y}} F_{y}(x, y, w) \\
& =-\eta_{x}^{\mathrm{T}} Q_{x} \eta_{x}+2 P_{n}^{x} \eta_{x} f_{n 1}^{x}-\eta_{y}^{\mathrm{T}} Q_{y} \eta_{y}+2 P_{m}^{y} \eta_{y} f_{m 1}^{y}
\end{aligned}
$$

where $P_{n}^{x}=\left[\begin{array}{lll}p_{n 1}^{x} & \cdots & p_{n, n+1}^{x}\end{array}\right]$ and $P_{m}^{y}=\left[\begin{array}{lll}p_{m 1}^{y} & \cdots & p_{m, m+1}^{y}\end{array}\right]$. 
Now, using the inequalities (15)-(20), obtain,

$$
\begin{aligned}
\left\|f_{n 1}^{x}\right\| & \leq \kappa_{f x}^{x}\|x\|+\kappa_{f x}^{y}\|y\| \\
\left\|f_{m 1}^{y}\right\| & \leq \kappa_{f y}^{x}\|x\|+\kappa_{f y}^{y}\|y\|
\end{aligned}
$$

where $\kappa_{f x}^{x}, \kappa_{f x}^{y}, \kappa_{f y}^{x}$ and $\kappa_{f y}^{y}$ are all positive constants.

Substituting (31) and (32) into (30), and using $\|x\| \leq\left\|\eta_{x}\right\|$ and $\|y\| \leq\left\|\eta_{y}\right\|$, obtain,

$$
\begin{aligned}
\dot{V}\left(\eta_{x}, \eta_{y}\right) & \leq-\left(\lambda_{m}\left(Q_{x}\right)-2 \kappa_{f x}^{x}\left\|P_{n}^{x}\right\|\right)\left\|\eta_{x}\right\|^{2}-\left(\lambda_{m}\left(Q_{y}\right)-2 \kappa_{f_{y}}^{y}\left\|P_{m}^{y}\right\|\right)\left\|\eta_{y}\right\|^{2}+2\left(\kappa_{f_{x}}^{y}\left\|P_{n}^{x}\right\|+\kappa_{f_{y}}^{x}\left\|P_{m}^{y}\right\|\right)\left\|\eta_{x}\right\|\left\|\eta_{y}\right\| \\
& =-\zeta^{\mathrm{T}} \Lambda \zeta .
\end{aligned}
$$

where $\zeta=\left[\left\|\eta_{x}\right\|\left\|\eta_{y}\right\|\right]^{\mathrm{T}},\left\|P_{n}^{x}\right\|=\left\|P_{n}^{x \varepsilon \alpha}\right\| \varepsilon_{\alpha x},\left\|P_{m}^{y}\right\|=\left\|P_{m}^{y \varepsilon \alpha}\right\| \varepsilon_{\alpha y}$ and

$$
\Lambda=\left[\begin{array}{cc}
\left(\lambda_{m}\left(Q_{x}\right)-2 \varepsilon_{\alpha x} \kappa_{f x}^{x}\left\|P_{n}^{x \varepsilon \alpha}\right\|\right) & -\left(\varepsilon_{\alpha x} \kappa_{f_{x}}^{y}\left\|P_{n}^{x \varepsilon \alpha}\right\|+\varepsilon_{\alpha y} \kappa_{f_{y}}^{x}\left\|P_{m}^{y \varepsilon \alpha}\right\|\right) \\
-\left(\varepsilon_{\alpha x} \kappa_{f_{x}}^{y}\left\|P_{n}^{x \varepsilon \alpha}\right\|+\varepsilon_{\alpha y} \kappa_{f_{y}}^{x}\left\|P_{m}^{y \varepsilon \alpha}\right\|\right) & \left(\lambda_{m}\left(Q_{y}\right)-2 \varepsilon_{\alpha y} \kappa_{f_{y}}^{y}\left\|P_{m}^{y \varepsilon \alpha}\right\|\right)
\end{array}\right] .
$$

By Theorems 1 and 2, and Propositions 1 and 2, obtain,

$$
\left\|P_{n}^{x}\right\|=\left\|P_{n}^{x \varepsilon \alpha}\right\| \varepsilon_{\alpha x} \rightarrow 0 \quad \forall \varepsilon_{\beta x} \in\left(0, \varepsilon_{\beta x}^{*}\right) \text { as } \varepsilon_{\alpha x} \rightarrow 0,\left\|P_{m}^{y}\right\|=\left\|P_{m}^{y \varepsilon \alpha}\right\| \varepsilon_{\alpha y} \rightarrow 0 \forall \varepsilon_{\beta y} \in\left(0, \varepsilon_{\beta y}^{*}\right) \text { as } \varepsilon_{\alpha y} \rightarrow 0 \text {. }
$$

Therefore, there exist $\varepsilon_{\alpha x}^{* *}$ and $\varepsilon_{\alpha y}^{* *}$ such that $\Lambda>0$ holds for all $0<\varepsilon_{\alpha x}<\varepsilon_{\alpha x}^{* *}$ and $0<\varepsilon_{\alpha y}<\varepsilon_{\alpha y}^{* *}$. Consequently, we have $\dot{V}\left(\eta_{x}, \eta_{y}\right) \leq 0$.

Using the fact that Lyapunov function $V\left(\eta_{x}, \eta_{y}\right)$ is a positive define function and its time derivative is a negative define function if $\Lambda>0$ holds, we conclude that the closed-loop system (27) is stable. In fact, $\dot{V}\left(\eta_{x}, \eta_{y}\right)=0$ means $x=y=0, \sigma_{x}=\sigma_{x 0}$ and $\sigma_{y}=\sigma_{y 0}$. By invoking LaSalle's invariance principle, it is obvious that the closed-loop system (27) is exponentially stable. As a result, we have the following theorem.

Theorem 3: Under Assumptions 1 and 2, if there exist the gains $\alpha_{i}^{x}, \beta_{j}^{x}, \alpha_{k}^{y}$ and $\beta_{l}^{y}$ such that the matrix $A_{x}$ for all $0<\varepsilon_{\beta x}<\varepsilon_{\beta x}^{*}$ and $0<\varepsilon_{\alpha x}<\varepsilon_{\alpha x}^{*}$, and the matrix $A_{y}$ for all $0<\varepsilon_{\beta y}<\varepsilon_{\beta y}^{*}$ and $0<\varepsilon_{\alpha y}<\varepsilon_{\alpha y}^{*}$ are all Hurwitz, and then $x=y=0, \sigma_{x}=\sigma_{x 0}$ and $\sigma_{y}=\sigma_{y 0}$ is an exponentially stable equilibrium point of the closed-loop system (27) for all $0<\varepsilon_{\beta x}<\varepsilon_{\beta x}^{*}, 0<\varepsilon_{\beta y}<\varepsilon_{\beta y}^{*}, 0<\varepsilon_{\alpha x}<\varepsilon_{\alpha x}^{* *}$ and $0<\varepsilon_{\alpha y}<\varepsilon_{\alpha y}^{* *}$. Moreover, if all assumptions hold globally, and then it is globally exponentially stable.

In the same way, for the case of $\varepsilon_{\alpha x}=\varepsilon_{\beta x}$ and $\varepsilon_{\alpha y}=\varepsilon_{\beta y}$, we have the following theorem.

Theorem 4: Under Assumptions 1 and 2, if there exist the gains $\alpha_{i}^{x}, \beta_{j}^{x}, \alpha_{k}^{y}$ and $\beta_{l}^{y}$ such that the matrix $A_{x}$ for all $0<\varepsilon_{\alpha x}=\varepsilon_{\beta x}<\varepsilon_{x}^{*}$, and the matrix $A_{y}$ for all $0<\varepsilon_{\alpha y}=\varepsilon_{\beta y}<\varepsilon_{y}^{*}$ are all Hurwitz, and then $x=y=0, \sigma_{x}=\sigma_{x 0}$ and $\sigma_{y}=\sigma_{y 0}$ is an exponentially stable equilibrium point of the closed-loop system (27) for all $0<\varepsilon_{\alpha x}=\varepsilon_{\beta x}<\varepsilon_{x}^{* *}$ and $0<\varepsilon_{\alpha y}=\varepsilon_{\beta y}<\varepsilon_{y}^{* *}$. Moreover, if all assumptions hold globally, and then it is globally exponentially stable.

\subsection{Synthetic Method}

The control laws $u_{x}$ and $u_{y}$ are taken as,

$$
\left\{\begin{array}{l}
u_{x}=-\varepsilon_{\alpha x}^{-1}\left(\alpha_{1}^{x} x_{1}+\alpha_{2}^{x} x_{2}+\cdots+\alpha_{n}^{x} x_{n}+\alpha_{n+m+1}^{x} \sigma_{x}+\alpha_{n+1}^{x} y_{1}+\alpha_{n+2}^{x} y_{2}+\cdots+\alpha_{n+m}^{x} y_{m}\right) \\
\dot{\sigma}_{x}=\varepsilon_{\beta x}^{-1}\left(\beta_{1}^{x} x_{1}+\beta_{2}^{x} x_{2}+\cdots+\beta_{n}^{x} x_{n}+\beta_{n+1}^{x} y_{1}+\beta_{n+2}^{x} y_{2}+\cdots+\beta_{n+m}^{x} y_{m}\right) \\
u_{y}=-\varepsilon_{\alpha y}^{-1}\left(\alpha_{1}^{y} x_{1}+\alpha_{2}^{y} x_{2}+\cdots+\alpha_{n}^{y} x_{n}+\alpha_{n+m+1}^{y} \sigma_{y}+\alpha_{n+1}^{y} y_{1}+\alpha_{n+2}^{y} y_{2}+\cdots+\alpha_{n+m}^{y} y_{m}\right) \\
\dot{\sigma}_{y}=\varepsilon_{\beta y}^{-1}\left(\beta_{1}^{y} x_{1}+\beta_{2}^{y} x_{2}+\cdots+\beta_{n}^{y} x_{n}+\beta_{n+1}^{y} y_{1}+\beta_{n+2}^{y} y_{2}+\cdots+\beta_{n+m}^{y} y_{m}\right)
\end{array}\right.
$$

where $\alpha_{i}^{x}, \alpha_{i}^{y}, \beta_{j}^{x}, \beta_{j}^{y}, \varepsilon_{\alpha x}, \varepsilon_{\alpha y}, \quad \varepsilon_{\beta x}$ and $\varepsilon_{\beta y}(i=1,2, \cdots n+m+1, j=1,2, \cdots n+m)$ are all positive constants. 
In the same way as Subsection 3.1, the closed-loop system can be rewritten as,

$$
\dot{\eta}=A \eta+F(x, y, w)
$$

where $\eta=\left[\begin{array}{llll}x^{\mathrm{T}} & y^{\mathrm{T}} & \sigma_{x}-\sigma_{x 0} & \sigma_{y}-\sigma_{y 0}\end{array}\right]^{\mathrm{T}}$,

$$
A=\left[\begin{array}{cccccccccc}
0 & 1 & \cdots & 0 & 0 & 0 & \cdots & 0 & 0 & 0 \\
0 & 0 & \ddots & 0 & 0 & 0 & \cdots & 0 & 0 & 0 \\
0 & 0 & \cdots & 1 & 0 & 0 & \cdots & 0 & 0 & 0 \\
-\varepsilon_{\alpha x}^{-1} \alpha_{1}^{x} & -\varepsilon_{\alpha x}^{-1} \alpha_{2}^{x} & \cdots & -\varepsilon_{\alpha x}^{-1} \alpha_{n}^{x} & -\varepsilon_{\alpha x}^{-1} \alpha_{n+1}^{x} & -\varepsilon_{\alpha x}^{-1} \alpha_{n+2}^{x} & \cdots & -\varepsilon_{\alpha x}^{-1} \alpha_{n+m}^{x} & -\varepsilon_{\alpha x}^{-1} \alpha_{n+m+1}^{x} & 0 \\
0 & 0 & \cdots & 0 & 0 & 1 & \cdots & 0 & 0 & 0 \\
0 & 0 & \cdots & 0 & 0 & 0 & \ddots & 0 & 0 & 0 \\
0 & 0 & \cdots & 0 & 0 & 0 & \cdots & 1 & 0 & 0 \\
-\varepsilon_{\alpha y}^{-1} \alpha_{1}^{y} & -\varepsilon_{\alpha y}^{-1} \alpha_{2}^{y} & \cdots & -\varepsilon_{\alpha y}^{-1} \alpha_{n}^{y} & -\varepsilon_{\alpha y}^{-1} \alpha_{n+1}^{y} & -\varepsilon_{\alpha y}^{-1} \alpha_{n+2}^{y} & \cdots & -\varepsilon_{\alpha y}^{-1} \alpha_{n+m}^{y} & 0 & -\varepsilon_{\alpha y}^{-1} \alpha_{n+m+1}^{y} \\
\varepsilon_{\beta x}^{-1} \beta_{1}^{x} & \varepsilon_{\beta x}^{-1} \beta_{2}^{x} & \cdots & \varepsilon_{\beta x}^{-1} \beta_{n}^{x} & \varepsilon_{\beta x}^{-1} \beta_{n+1}^{x} & \varepsilon_{\beta x}^{-1} \beta_{n+2}^{x} & \cdots & \varepsilon_{\beta x}^{-1} \beta_{n+m}^{x} & 0 & 0 \\
\varepsilon_{\beta y}^{-1} \beta_{1}^{y} & \varepsilon_{\beta y}^{-1} \beta_{2}^{y} & \cdots & \varepsilon_{\beta y}^{-1} \beta_{n}^{y} & \varepsilon_{\beta y}^{-1} \beta_{n+1}^{y} & \varepsilon_{\beta y}^{-1} \beta_{n+2}^{y} & \cdots & \varepsilon_{\beta y}^{-1} \beta_{n+m}^{y} & 0 & 0
\end{array}\right]
$$

and $F(x, y, w)$ is an $n+m+2 \times 1$ matrix, all its elements are equal to zero except for

$$
\begin{aligned}
f_{n 1} & =f_{x}(x, y, w)-f_{x}(0,0, w)-\left[g_{x}(x, y, w)-g_{x}(0,0, w)\right] f_{x}(0,0, w) g_{x}^{-1}(0,0, w) \text { and } \\
f_{n+m, 1} & =f_{y}(x, y, w)-f_{y}(0,0, w)-\left[g_{y}(x, y, w)-g_{y}(0,0, w)\right] f_{y}(0,0, w) g_{y}^{-1}(0,0, w) .
\end{aligned}
$$

Moreover, by the same way as Subsection 3.1, the functions $g_{x}(x, y, w)$ and $g_{y}(x, y, w)$ are integrated into $\varepsilon_{\alpha x}$ and $\varepsilon_{\alpha y}$, respectively.

The matrix $A$ can be designed to be Hurwitz for all $0<\varepsilon_{\beta x}<\varepsilon_{\beta x}^{*}, 0<\varepsilon_{\alpha x}<\varepsilon_{\alpha x}^{*}, 0<\varepsilon_{\beta y}<\varepsilon_{\beta y}^{*}$ and $0<\varepsilon_{\alpha y}<\varepsilon_{\alpha y}^{*}$. Thus, a quadratic Lyapunov function,

$$
V(\eta)=\eta^{\mathrm{T}} P \eta
$$

can be obtained. Where $P$ is the solution of Lyapunov equation $P A+A^{\mathrm{T}} P=-Q$ with any given positive define symmetric matrix $Q$.

Thus, using $V(\eta)$ as Lyapunov function candidate, and then its time derivative along the trajectories of the closed-loop systems (35) is,

$$
\dot{V}(\eta)=\eta^{\mathrm{T}}\left(P A+A^{\mathrm{T}} P\right) \eta+\frac{\partial V(\eta)}{\partial \eta} F(x, y, w)=-\eta^{\mathrm{T}} Q \eta+2 P_{n} \eta f_{n 1}+2 P_{n+m} \eta f_{n+m, 1}
$$

where

$$
P_{n}=\left[\begin{array}{llllll}
p_{n 1} & \cdots & p_{n n} & p_{n, n+1} & \cdots & p_{n, n+m+2}
\end{array}\right]
$$

and

$$
P_{n+m}=\left[\begin{array}{llllll}
p_{n+m, 1} & \cdots & p_{n+m, n} & p_{n+m, n+1} & \cdots & p_{n+m, n+m+2}
\end{array}\right] .
$$

Now, using the inequalities (15)-(20), obtain,

$$
\begin{aligned}
& \left\|f_{n 1}\right\| \leq \boldsymbol{\kappa}_{f x}^{x}\|x\|+\kappa_{f x}^{y}\|y\| \\
& \left\|f_{n+m, 1}\right\| \leq \boldsymbol{\kappa}_{f y}^{x}\|x\|+\kappa_{f y}^{y}\|y\|
\end{aligned}
$$

where $\kappa_{f x}^{x}, \kappa_{f x}^{y}, \kappa_{f y}^{x}$ and $\kappa_{f y}^{y}$ are all positive constants.

Substituting (38) and (39) into (37), and using $\|x\| \leq\|\eta\|$ and $\|y\| \leq\|\eta\|$, obtain,

$$
\begin{aligned}
\dot{V}(\eta) & \leq-\lambda_{m}(Q)\|\eta\|^{2}+2\left(\kappa_{f_{x}}^{x}\|x\|+\kappa_{f_{x}}^{y}\|y\|\right)\left\|P_{n}\right\|\|\eta\|+2\left(\kappa_{f_{y}}^{x}\|x\|+\kappa_{f_{y}}^{y}\|y\|\right)\left\|P_{n+m}\right\|\|\eta\| \\
& \leq-\left(\lambda_{m}(Q)-2\left(\kappa_{f_{x}}^{x}+\kappa_{f_{x}}^{y}\right)\left\|P_{n}^{\varepsilon \alpha}\right\| \varepsilon_{\alpha x}-2\left(\kappa_{f_{y}}^{x}+\kappa_{f_{y}}^{y}\right)\left\|P_{n+m}^{\varepsilon \alpha}\right\| \varepsilon_{\alpha y}\right)\|\eta\|^{2} .
\end{aligned}
$$


where $\left\|P_{n}\right\|=\left\|P_{n}^{\varepsilon \alpha}\right\| \varepsilon_{\alpha x}$ and $\left\|P_{n+m}\right\|=\left\|P_{n+m}^{\varepsilon \alpha}\right\| \varepsilon_{\alpha y}$.

By Theorems 1 and 2, and Propositions 1 and 2, obtain,

$$
\begin{gathered}
\left\|P_{n}\right\|=\left\|P_{n}^{\varepsilon \alpha}\right\| \varepsilon_{\alpha x} \rightarrow 0 \quad \forall \varepsilon_{\beta x} \in\left(0, \varepsilon_{\beta x}^{*}\right) \text { as } \varepsilon_{\alpha x} \rightarrow 0, \\
\left\|P_{n+m}\right\|=\left\|P_{n+m}^{\varepsilon \alpha}\right\| \varepsilon_{\alpha y} \rightarrow 0 \quad \forall \varepsilon_{\beta y} \in\left(0, \varepsilon_{\beta y}^{*}\right) \text { as } \varepsilon_{\alpha y} \rightarrow 0
\end{gathered}
$$

Thus, there exist $\varepsilon_{\alpha x}^{* *}$ and $\varepsilon_{\alpha y}^{* *}$ such that

$$
\lambda_{m}(Q)>2\left(\kappa_{f_{x}}^{x}+\kappa_{f_{x}}^{y}\right)\left\|P_{n}^{\varepsilon \alpha}\right\| \varepsilon_{\alpha x}+2\left(\kappa_{f_{y}}^{x}+\kappa_{f_{y}}^{y}\right)\left\|P_{n+m}^{\varepsilon \alpha}\right\| \varepsilon_{\alpha y}
$$

holds for all $0<\varepsilon_{\alpha x}<\varepsilon_{\alpha x}^{* *}$ and $0<\varepsilon_{\alpha y}<\varepsilon_{\alpha y}^{* *}$. Consequently, we have $\dot{V}(\eta) \leq 0$.

Using the fact that Lyapunov function $V(\eta)$ is a positive define function and its time derivative is a negative define function if the inequality (41) holds, we conclude that the closed-loop system (35) is stable. In fact, $\dot{V}(\eta)=0$ means $x=y=0, \sigma_{x}=\sigma_{x 0}$ and $\sigma_{y}=\sigma_{y 0}$. By invoking LaSalle's invariance principle, it is easy to know that the closed-loop system (35) is exponentially stable. As a result, the following theorem can be established.

Theorem 5: Under Assumptions 1 and 2, if there exist the gains $\alpha_{i}^{x}, \alpha_{i}^{y}, \beta_{j}^{x}$ and $\beta_{j}^{y}$ such that the matrix $A$ is Hurwitz for all $0<\varepsilon_{\beta x}<\varepsilon_{\beta x}^{*}, 0<\varepsilon_{\alpha x}<\varepsilon_{\alpha x}^{*}, 0<\varepsilon_{\beta y}<\varepsilon_{\beta y}^{*}$ and $0<\varepsilon_{\alpha y}<\varepsilon_{\alpha y}^{*}$, and then $x=y=0$, $\sigma_{x}=\sigma_{x 0}$ and $\sigma_{y}=\sigma_{y 0}$ is an exponentially stable equilibrium point of the closed-loop system (35) for all for all $0<\varepsilon_{\beta x}<\varepsilon_{\beta x}^{*}, 0<\varepsilon_{\beta y}<\varepsilon_{\beta y}^{*}, 0<\varepsilon_{\alpha x}<\varepsilon_{\alpha x}^{* *}$ and $0<\varepsilon_{\alpha y}<\varepsilon_{\alpha y}^{* *}$. Moreover, if all assumptions hold globally, then it is globally exponentially stable.

In the same way, for the case of $\varepsilon_{\alpha x}=\varepsilon_{\beta x}$ and $\varepsilon_{\alpha y}=\varepsilon_{\beta y}$, we have the following theorem.

Theorem 6: Under Assumptions 1 and 2, if there exist the gains $\alpha_{i}^{x}, \alpha_{i}^{y}, \beta_{j}^{x}$ and $\beta_{j}^{y}$ such that the matrix $A$ is Hurwitz for all $0<\varepsilon_{\alpha x}=\varepsilon_{\beta x}<\varepsilon_{x}^{*}$ and $0<\varepsilon_{\alpha y}=\varepsilon_{\beta y}<\varepsilon_{y}^{*}$, and then $x=y=0, \sigma_{x}=\sigma_{x 0}$ and $\sigma_{y}=\sigma_{y 0}$ is an exponentially stable equilibrium point of the closed-loop system (35) for all $0<\varepsilon_{\alpha x}=\varepsilon_{\beta x}<\varepsilon_{x}^{* *}$ and $0<\varepsilon_{\alpha y}=\varepsilon_{\beta y}<\varepsilon_{y}^{* *}$. Moreover, if all assumptions hold globally, then it is globally exponentially stable.

Discussion 6: From Decomposition and Synthetic methods above, it is obvious that: 1) although they are developed with two variable systems, it is not hard to extend them to the multiple variable systems; 2 ) as the subsystems increase, Decomposition method is simpler and more practical than Synthetic method since we can design the controllers for every subsystems, respectively, and then combine them such that the whole closed-loop system is asymptotically stable; 3) for designing a high performance controller, Synthetic method is more excellent than Decomposition method since we can use all the state variables to design the controller and integrator.

Discussion 7: From the procedure of stability analysis above, it is obvious that so long as the bounded conditions (13)-(20) are satisfied, the asymptotically stable control can be achieved. This shows that the striking feature of linear general integral control, that is, its robustness with respect to $f_{x}(x, y, w), g_{x}(x, y, w), f_{y}(x, y, w)$ and $g_{y}(x, y, w)$, is clearly demonstrated by Equal ratio gain technique. Therefore, Equal ratio gain technique is a powerful tool to solve the control design problem of uncertain nonlinear system, and then makes the engineers more easily design a stable controller. Moreover, for the 2-order system, linear general integral control can be reduced to PID control. Thus, Equal ratio gain technique can clearly explain the reason why PID control has good robustness, too.

Discussion 8: Form all the statements of Sections 3 and 4, it is not hard to see that although Equal ratio gain technique is demonstrated by a class of special system and linear general integral control, its application is not limited in them and can be extend to solve the other relevant problem since Routh's stability criterion, Lyapunov equation and Lyapunov method are all universal. For examples: 1) if the system is not given in the form (10), one can find a transformation matrix that takes the given system to this form if the system is controllable; 2) by combining Equal ratio gain technique with Feedback linearization technique, we can achieve the design of nonlinear integral controller; 3 ) as the integrator gains are equal to zero, the control is reduced to proportional control, and the similar conclusions can still be obtained.

Discussion 9: Although the design procedure above looks quite complicated, there need not abstruse theory since Routh's stability criterion, Lyapunov equation and Lyapunov method are all simple enough to be pre- 
sented in the text book and practical enough to have been used in the real-word problem. Therefore, Equal ratio gain technique has not only the important theoretical significance but also the broad application prospects.

\section{Example and Simulation}

Consider the pendulum system [1] described by,

$$
\ddot{\theta}=-a \sin (\theta)-b \dot{\theta}+c T
$$

where $a, b, c>0, \theta$ is the angle subtended by the rod and the vertical axis, and $T$ is the torque applied to the pendulum. View $T$ as the control input and suppose we want to regulate $\theta$ to $\delta$. Now, taking $x_{1}=\theta-\delta, \dot{x}_{2}=\dot{\theta}$, the pendulum system can be written as,

$$
\left\{\begin{array}{l}
\dot{x}_{1}=x_{2} \\
\dot{x}_{2}=-a \sin \left(x_{1}+\delta\right)-b x_{2}+c u
\end{array}\right.
$$

and then it can be verified that $u_{0}=a \sin (\delta) / c$ is the steady-state control that is needed to maintain equilibrium at the origin and the control law is,

$$
\left\{\begin{array}{l}
u=-\varepsilon_{\alpha}^{-1}\left(\alpha_{1} x_{1}+\alpha_{2} x_{2}+\alpha_{3} \sigma\right) \\
\dot{\sigma}=\varepsilon_{\beta}^{-1}\left(\beta_{1} x_{1}+\beta_{2} x_{2}\right)
\end{array}\right.
$$

Thus, the closed-loop system can be written as,

$$
\dot{\eta}=A \eta+F(x, w)
$$

where

$$
\begin{gathered}
\eta=\left[\begin{array}{lll}
x_{1} & x_{2} & \sigma-\sigma_{0}
\end{array}\right]^{\mathrm{T}}, \quad F(x, w)=\left[\begin{array}{ccc}
0 & a \sin (\delta)-a \sin \left(x_{1}+\delta\right) & 0
\end{array}\right]^{\mathrm{T}} \text { and } \\
A=\left[\begin{array}{ccc}
0 & 1 & 0 \\
-\varepsilon_{\alpha}^{-1} c \alpha_{1} & -\varepsilon_{\alpha}^{-1} c\left(\alpha_{2}+\varepsilon_{\alpha} c^{-1} b\right) & -\varepsilon_{\alpha}^{-1} c \alpha_{3} \\
\varepsilon_{\beta}^{-1} \beta_{1} & \varepsilon_{\beta}^{-1} \beta_{2} & 0
\end{array}\right] .
\end{gathered}
$$

The normal parameters are $a=c=10$ and $b=2$, and in the perturbed case, $b$ and $c$ are reduced to 1 and 5, respectively, corresponding to double the mass. Thus, we have, $\left\|a \sin (\delta)-a \sin \left(x_{1}+\delta\right)\right\| \leq 10\|\eta\|$.

Now, if the gains are taken as $\alpha_{1}=8, \alpha_{2}=5, \alpha_{3}=9, \beta_{1}=5$ and $\beta_{2}=3$, and then with $a=10, c=5$ and $b=1$, the following inequality,

$$
c \alpha_{2} \alpha_{3} \beta_{2}+\varepsilon_{\alpha}\left(c \alpha_{2} \alpha_{1}+b \alpha_{3} \beta_{2}-\alpha_{3} \beta_{1}\right)+\varepsilon_{\alpha} \varepsilon_{\alpha} b \alpha_{1}>0
$$

holds for all $0<\varepsilon_{\beta}=\varepsilon_{\alpha}<\infty$, and then the matrix $A$ is Hurwitz for all $0<\varepsilon_{\beta}=\varepsilon_{\alpha}<\infty$. Therefore, we have, $\varepsilon_{\beta}^{*}=\varepsilon_{\alpha}^{*}=\infty$.

By solving the Lyapunov equation $P A+A^{\mathrm{T}} P=-I$ with $c=5, b=1$ and $\varepsilon_{\beta}=\varepsilon_{\alpha}$, obtain,

$$
20\left\|P_{2}\right\|<1 \quad \forall 0<\varepsilon_{\beta}=\varepsilon_{\alpha} \leq \varepsilon_{\beta}^{* *}=\varepsilon_{\alpha}^{* *}=1.28
$$

Thus, the asymptotical stability of the closed-loop system can be ensured for all $0<\varepsilon_{\beta}=\varepsilon_{\alpha} \leq 1.28$. Consequently, taking $\varepsilon_{\beta}=\varepsilon_{\alpha}=1.28$, the simulation is implemented under the normal and perturbed cases, respectively. Moreover, in the perturbed case, we consider an additive impulse-like disturbance $\mathrm{d}(t)$ of magnitude 80 acting on the system input between $15 \mathrm{~s}$ and $16 \mathrm{~s}$.

Figure 1 showed the simulation results under normal (solid line) and perturbed (dashed line) cases. The following observations can be made: the system responses under the normal and perturbed cases are almost identical before the additive impulse-like disturbance appears. From the simulation results and design procedure, it is obvious that by Equal ratio gain technique, we can tune a linear general integral controller with good robustness. This demonstrates that not only linear general integral control can effectively deal with the uncertain nonlinearity but also Equal ratio gain technique is a powerful tool to solve the control design problem of uncertain nonli- 


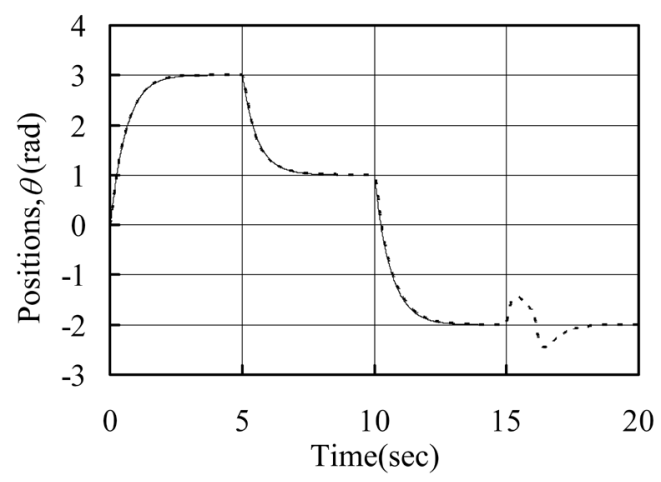

Figure 1. System output under normal (solid line) and perturbed (dashed line) cases.

near system, and then makes the engineers more easily design a stable controller. Consequently, Equal ratio gain technique has not only the important theoretical significance but also the broad application prospects.

\section{Conclusions}

In conjunction with linear general integral control, this paper proposes a fire-new control design technique, named Equal ratio gain technique, and then develops two kinds of control design methods, that is, Decomposition and Synthetic methods, for a class of uncertain nonlinear system. The main conclusions are as follows: 1) a canonical system matrix can be designed to be always Hurwitz as any row controller gains, or controller and its integrator gains increase with the same ratio; 2 ) as any row controller gains, or controller and its integrator gains of a canonical system matrix tend to infinity with the same ratio, if it is always Hurwitz, and then the same row solutions of Lyapunov equation all tend to zero; 3) theorems to ensure semi-globally asymptotic stability are established in terms of some bounded information. Moreover, the striking robustness of linear general integral control and PID control is clearly illustrated by Equal ratio gain technique. All these mean that Equal ratio gain technique is a powerful tool to solve the control design problem of uncertain nonlinear system, and then makes the engineers more easily design a stable controller. Consequently, Equal ratio gain technique has not only the important theoretical significance but also the broad application prospects.

These conclusions above are further confirmed by the design example and simulation results.

\section{References}

[1] Khalil, H.K. (2007) Nonlinear Systems. 3rd Edition, Electronics Industry Publishing, Beijing, 449-453, 551.

[2] Liu, B.S. and Tian, B.L. (2012) General Integral Control Design Based on Linear System Theory. Proceedings of the 3rd International Conference on Mechanic Automation and Control Engineering, 5, 3174-3177.

[3] Liu, B.S. and Tian, B.L. (2012) General Integral Control Design Based on Sliding Mode Technique. Proceedings of the 3rd International Conference on Mechanic Automation and Control Engineering, 5, 3178-3181.

[4] Liu, B.S., Li, J.H. and Luo, X.Q. (2014) General Integral Control Design via Feedback Linearization. Intelligent Control and Automation, 5, 19-23. http://dx.doi.org/10.4236/ica.2014.51003

[5] Liu, B.S., Luo, X.Q. and Li, J.H. (2014) General Integral Control Design via Singular Perturbation Technique. International Journal of Modern Nonlinear Theory and Application, 3, 173-181. http://dx.doi.org/10.4236/ijmnta.2014.34019

[6] Liu, B.S., Luo, X.Q. and Li, J.H. (2013) General Concave Integral Control. Intelligent Control and Automation, 4, 356361. http://dx.doi.org/10.4236/ica.2013.44042

[7] Liu, B.S., Luo, X.Q. and Li, J.H. (2014) General Convex Integral Control. International Journal of Automation and Computing, 11, 565-570. http://dx.doi.org/10.1007/s11633-014-0813-6

[8] Liu, B.S. (2014) Constructive General Bounded Integral Control. Intelligent Control and Automation, 5, 146-155. http://dx.doi.org/10.4236/ica.2014.53017

[9] Liu, B.S. (2014) On the Generalization of Integrator and Integral Control Action. International Journal of Modern Nonlinear Theory and Application, 3, 44-52. http://dx.doi.org/10.4236/ijmnta.2014.32007

[10] Gajic, Z. (1995) Lyapunov Matrix Equation in System Stability and Control. Mathematics in Science and Engineering, 195, 30-31. 
Scientific Research Publishing (SCIRP) is one of the largest Open Access journal publishers. It is currently publishing more than 200 open access, online, peer-reviewed journals covering a wide range of academic disciplines. SCIRP serves the worldwide academic communities and contributes to the progress and application of science with its publication.

Other selected journals from SCIRP are listed as below. Submit your manuscript to us via either submit@scirp.org or Online Submission Portal.
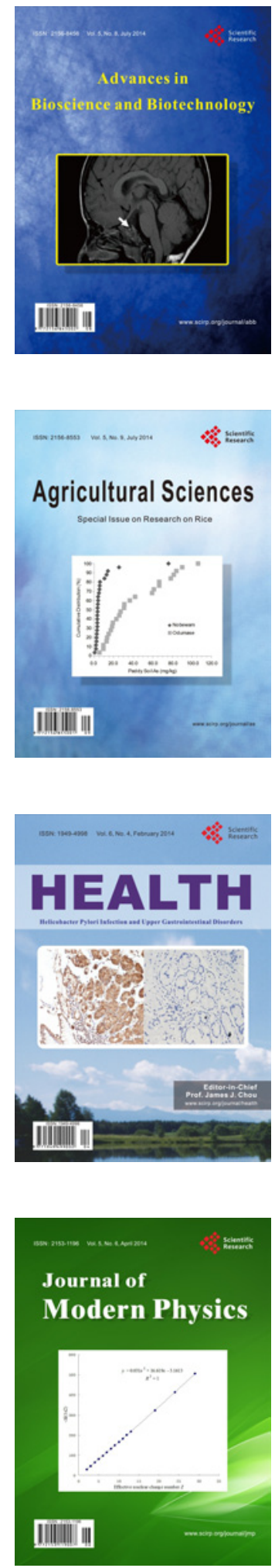
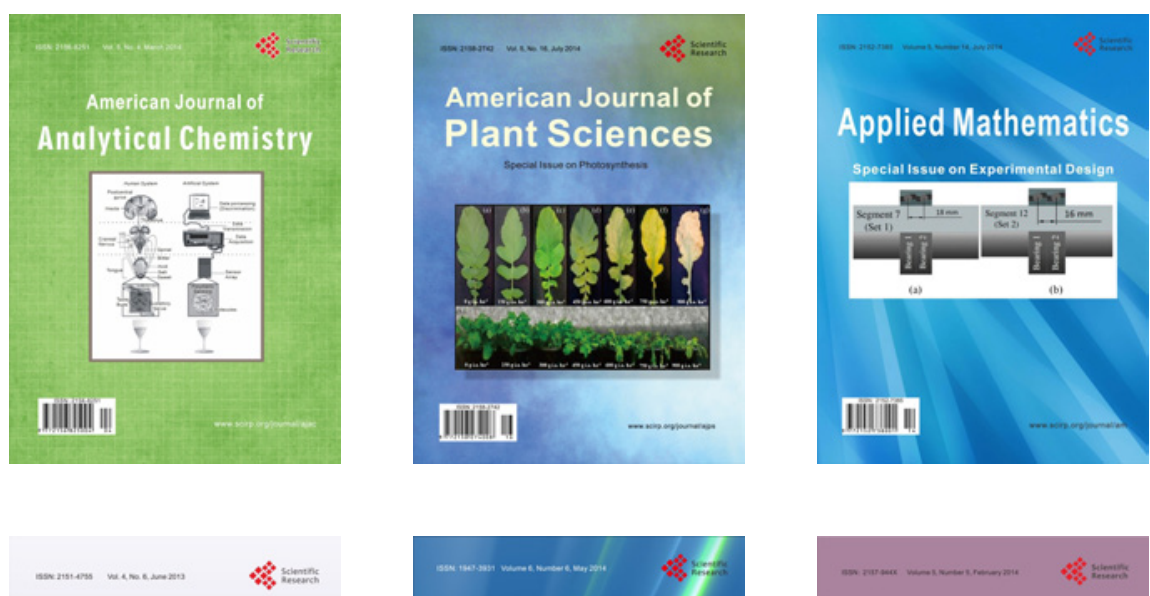

Creative Education
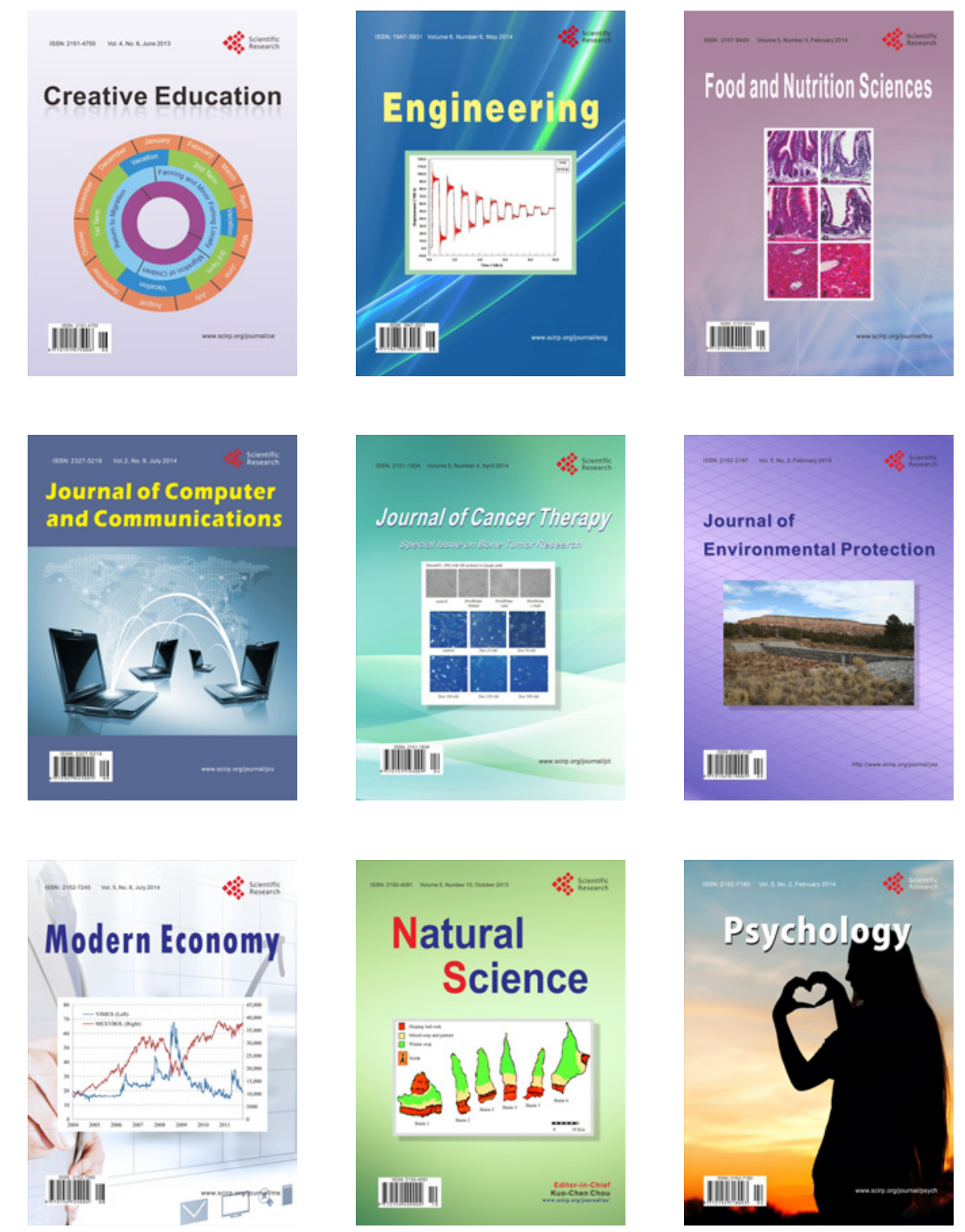